\title{
Les Crustacés du réservoir de la Fontaine des Suisses à Dijon
}

\author{
Par Bernard Dussarti1), François Graf ${ }^{2}$ ) et Roger Husson ${ }^{2}$ )
}

Avec planches 50 (1) - 52 (3)

\section{Préambule}

La Fontaine des Suisses est actuellement enclavée dans l'Hospice des vieillards de Champmaillot, c'est à dire qu'elle se trouve maintenant dans l'agglomération même de Dijon.

Elle s'appelait autrefois source de Champmaillot, l'eau en était captée et coulait en canalisations souterraines qui dès le XVe siècle alimentaient six fontaines à Dijon.

Elle doit son nom actuel de Fontaine des Suisses au fait que, sur le plateau où elle prend naissance, 30.000 Suisses sous les ordres de Jacques de Watteville, campaient, quand assiègeant Dijon en septembre 1513, ils avaient installé là les batteries qui devaient bombarder la ville. Le siège ayant été levé, Dijon fut épargné et les Suisses s'en allèrent en laissant seulement leur nom à cette fontaine qui alimenta encore la ville en eau semi-potable pendant trois siècles, jusqu'à l'établissement du système d'adduction d'eau créé par Darcy en 1840. Actuellement les eaux de la Fontaine des Suisses servent seulement aux travaux de voirie.

Nous devons remercier le service des eaux de la ville de Dijon, qui, lors d'une opération de vidange du réservoir, a bien voulu autoriser une descente dans le dit réservoir, ce qui permit d'y pêcher en grand nombre les Crustacés Copépodes et Amphipodes qui font l'objet de cette note.

1) Centre de Recherches hydrobiologiques du C.N.R.S., Gif sur Yvette-91.

$\left.{ }^{2}\right)$ Laboratoire de Biologie Générale, Faculté des Sciences, 2 Boulevard Gabriel. 21 - Dijon. 


\section{COPÉPODES}

Par B. Dussart

Dans les prélèvements effectués ont été observées les espèces de Copépodes suivantes :

Macrocyclops albidus (Jurine)

Eucyclops serrulatus (Fischer)

Eucyclops serrulatus var. mihi

Acanthocyclops venustus (Norman et Scott)

Acanthocyclops vernalis (Fischer)

Acanthocyclops robustus (G.O. Sars)

Ces espèces appellent quelques remarques:

\section{Macrocyclops albidus}

Cette grande espèce s'adapte à de nombreux milieux. Elle est particulièrement fréquente dans les grandes mares à eaux claires et végétation abondante. Sténotherme d'eau froide (Roy 1922), elle a une prédilection pour les eaux se renouvelant lentement. Il n'est donc pas anormal de la trouver à la Fontaine des Suisses. Cosmopolite, elle a été observée un peu partout en France comme dans toute l'Europe, en Amérique du Nord et du Sud, en Asie et même aux Iles Hawaï !

\section{Eucyclops serrulatus}

Autre forme cosmopolite dont la malléabilité est bien connue. Elle a été trouvée ici sous la forme typique et sous une forme légèrement différente du type (fig. 1-6). La $\mathrm{P}_{5}$ de cette dernière possède des soies longues et surtout une épine peu épaisse à la base et élancée. Chez un individu, la soie médiane (terminale) présentait une malformation lui donnant un aspect bifide (fig. 5).

Chez l'espèce type, les caractéristiques des individus sont (fig. 1-4) : Longueur (sans les soies furcales) : $1120 \mu$

$A_{1}$ atteignant la suture $T h_{2}-T h_{3}$

Rapport L/l de la furca : 3,8

Rapport Ti/Te : 1,67

Rapport des épines de l'endopodite 3 de $\mathrm{P}_{4}: 1,39$

L'espèce non conforme au type avait pour caractéristiques essentielles (fig. 5) :

Rapport L/l de la furca : 5,1

Rapport Ti/Te : 1,40

Rapport des épines de l'endopodite 3 de $\mathrm{P}_{4}: 1,47$

Les différences existant entre ces deux formes ne sont pas suffi santes pour désigner d'un nom la variété à épine de $\mathrm{P}_{5}$ élancée. Il me 
suffit de mentionner son existence qui la rapproche, semble-t-il, de $E$. porrectus que Kiefer (1932) a décrite de Yougoslavie, mais dont la furca est beaucoup plus élancée.

\section{Acanthocyclops venustus}

Cette espèce est réputée rare (fig. 7,8 ). Elle a été trouvée en Suisse par Graeter (1908), en Allemagne par Klie (1928), en Angleterre par Gurney (1933), en Belgique par Kiefer (1936). Herbst (1954) l'a retrouvée en Allemagne et Lindberg (1949) en Suède.

En France, elle a été découverte par Hertzog (1930) à Strasbourg. Je l'ai retrouvée récemment dans la grotte de la Balme (Isère) où j'ai observé à la fois des mâles et des femelles.

Cette espèce «fugitive " au sens de Hutchinson (1951), bien que rare parce que troglophile sous nos latitudes, n'en existe pas moins dans les mousses (Sphaignes) en Allemagne du Nord (Kiefer 1960) et en Suède (Lindberg 1949).

\section{Acanthocyclops vernalis et Acanthocyclops robustus}

L'existence de ces deux espèces dans un même milieu aussi confiné que la Fontaine des Suisses est également à signaler. On sait que ces deux formes ne diffèrent l'une de l'autre que par quelques caractères.

Si la position systématique des espèces dénommées par Gurney « du groupe vernalis", ne pose pas de question, il n'en est pas de même de la parenté existant entre $A$. vernalis et $A$. robustus, et de la variabilité de chacune de ces deux espèces. Déjà Sars (1918), s'il séparait nettement robustus des autres formes du groupe vernalis, rassemblait sous le nom de $C$. lucidulus, le $C$. vernalis de Fischer et une variété qu'il n'a pas dénommée et ayant des caractères intermédiaires entre $C$. vernalis et $C$. robustus.

Lindberg (1953), critiquant les expériences de Lowndes (1928), constate que la forme robustus est plus constante dans la nature que sa voisine vernalis. Se basant sur les caractères primitifs de la première de ces formes, il préfère donner au type le nom de $C$. robustus et à l'espèce à épines moins nombreuses donc plus évoluée, le nom de $C$. vernalis. Malgré les arguments forts logiques donnés par cet auteur, il ne semble pourtant pas qu'il soit possible de le suivre, la dénomination C.vernalis Fischer datant de 1852, tandis que $C$. robustus Sars n'ayant été décrite qu'en 1863, époque où la formule des épines des $\operatorname{Exp}_{3}$ n'était pas encore prise en considération.

Malgré l'apparente variabilité des populations, il semble en fait prouvé que ces espèces sont à conserver, $C$. robustus étant plus stable, $C$. vernalis étant au contraire beaucoup plus malléable. Il serait 
bon que les formes de Thallwitz (1926) soient reprises en considération et qu'une étude statistique de populations naturelles soit entreprise pour apprécier la valeur systématique de ces deux «sous espèces" en formation, aussi «nettement» caractérisées que les «espèces» du genre Cyclops s. str. par exemple.

Rappelons que pour Thallwitz il y aurait parallélisme entre

$A$. robustus setiger et $A$. vernalis typica

A. robustus insueta et $A$. vernalis infesta

et enfin entre

A. robustus armata et $A$. vernalis aculeata.

Les exemplaires de $C$. robustus trouvés ici avaient pour caractéristiques :

$\mathrm{L}: 1440 \mu$

Rapport L/l de la fusca : 6,5 !

Rapport Ti/Te : 1,61

Rapport des épines de l'endopodite 3 de $\mathrm{P}_{4}: 1,00$.

Nous ne pourrons pas malheureusement et à défaut de matériel en quantité suffisante trancher statistiquement la question.

Sur d'autres populations ce travail est en cours, mais déjà la simple coexistence de ces deux formes dans un milieu aussi réduit que la Fontaine des Suisses laisse à penser que nous avons bien là à faire à deux espèces bien distinctes. Nous rejoignons ainsi les conclusions prudentes de Kiefer (1960) bien que Petkovski (1954) ait estimé que les trois formes qu'il a étudié de Yougoslavie (vernalis, robustus, setiger) ne puissent être séparées et doivent être rassemblées dans l'unique appellation de $A$. vernalis. Thiébaud (1953) a également trouvé les deux espèces dans le même milieu du Meienriederloch près de Bienne (Suisse) et considère les deux espèces comme parfaitement valables. Ses figures ne permettent malheureusement pas de dire quelles formes il a observés, ni s'il y a correspondance étroite entre son $A$. robustus et le nôtre.

Pour notre part, nous pensons qu'il y a un certain nombre de formes dont la description doit être à nouveau précisée et dont la distribution est encore mal connue. Sans contester la manifeste variabilité de ces formes, il n'en existe pas moins des types.

La figure 11 (a-a') montre sur un exemplaire de lagune espagnole (Las Marismas) récemment explorée (Dussart 1964) une anomalie fréquente: la dissymétrie congénitale (?) : l'endopodite 3 de la quatrième patte possède à droite deux soies internes et à gauche une seule.

Cette même figure permet d'affirmer que les individus récoltés à l'étang du Plessis (Loir et Cher) (fig. 14) et à la Fontaine des Suisses à Dijon (fig. 12) sont du même type et ressemblent à Acanthocyclops 
robustus var. aphanes, tandis qu'en Camargue (fig. 13) comme aux Marismas ${ }^{3}$ ) (fig. 11) donc dans les mêmes conditions écologiques cette fois, c'est la var. setiger qui se manifeste. En Camargue, à côté de cette variété existe une autre forme plus robuste (fig. 9), aux épines plus fortes et relativement plus courtes, aux soies non allongées que nous n'identifierons pas, tandis que la variété du Lac d'Eguzon (fig. 10) se caractérise par une forme plus grêle, rappelant un peu la variété setiger sans en avoir les soies très allongées. On remarque également que la transformation de la soie externe de l'endopodite 3 de $\mathrm{P}_{4}$ s'accompagne toujours d'un déplacement de son insertion vers l'extrémité de l'article. Ces deux caractères associés ne peuvent pas être considérés à priori comme de simples fluctuations individuelles d'autant plus qu'ils correspondent à des épines terminales subégales. Les six formes présentées ici et provenant de cinq milieux différents se ramènent donc finalement à deux types: le type setiger et un type trispinosa mihi.

Comme il a été dit plus haut, il faudra attendre le résultat des études en cours pour définir si ces variétés sont de bonnes formes, de vraies sous-espèces ou de simples fluctuants écologiques.

En résumé et pour permettre sans attendre, une détermination suffisamment précise des espèces et variétés de ce genre, la clé provisoire suivante est présentée :

\section{Genre Acanthocyclops Kiefer 1927}

Opercule anal parfois très développé; branches de la furca sans carène; réceptacle séminal formé de deux parties dont l'antérieure plutôt développée et la postérieure constituant deux cornes plus ou moins étirées latéralement. Antennules sans membrane hyaline aux derniers articles.

$\mathrm{P}_{1}$ à $\mathrm{P}_{4}$ à exopodite 3 muni de 7 ou de 8 soies ou épines.

$\mathrm{P}_{5}$ biarticulée ; article basilaire plus ou moins élargi ; article terminal portant une soie terminale bien développée et une épine le plus souvent réduite, insérée dans la deuxième moitié de l'article et généralement subapicale.

Deux sous-genres: Megacyclops Claus 1893 et Acanthocyclops s. str. Kiefer 1927 qui se différencient essentiellement par les branches furcales respectivement ciliées au bord interne ou non et par l'article basilaire de $\mathrm{P}_{5}$ fortement élargi ou non.

3) Cette forme a été simplement nommée $A$. robustus dans la note précédemment citée afin d'éviter de poser un problème taxinomique délicat que la note présente ne veut pas davantage résoudre définitivement. 
Le sous-genre Megacyclops est représenté en France essentiellement par l'espèce Megacyclops viridis Jurine et sa forme néoténique clausi; cependant les deux autres espèces $M$. gigas et $M$. latipes devraient être recherchées.

\section{Sous-Genre Megacyclops}

1 (2) Antennules de 17 articles chez la femelle .............. 3

2 (1) Antennules de 11 articles, branches des pattes à deux articles au lieu de trois; le reste des caractères comme $\boldsymbol{M}$. viridis viridis .... ...................... Megacyclops viridis clausi Lowndes

3 (4) Branches de la furca relativement courtes, au plus quatre fois plus longues que larges; soie terminale interne plus longue qu'une branche de la furca et au moins deux fois plus longue que la soie terminale externe ...........Megacyclops viridis viridis (Jurine)

4 (3) Branches de la furca au moins cinq fois plus longues que larges; soie terminale interne plus courte qu'une branche de la furca et un peu plus longue seulement que la soie terminale externe ..... 5

5 (6) Article terminal de l'endopodite de $\mathrm{P}_{4}$ élancé, plus de deux fois aussi long que large .....................

6 (5) Article terminal de l'endopodite de $\mathrm{P}_{4}$ ramassé, moins de deux fois aussi long que large ............Megacyclops latipes (Lowndes)

\section{Sous-Genre Acanthocyclops}

1 (2) Antennules à 17 articles chez la femelle ............. 3

2 (1) Antennules à moins de 17 articles chez la femelle ........ 7

3 (4) Réceptacle séminal papilloniforme; branches furcales 2,5 fois plus longues que larges; soie terminale interne plus de deux fois aussi longue que l'externe; épines apicales de l'endopodite de $\mathrm{P}_{4}$ très inégales, l'interne la plus longue; espèce d'eaux souterraines .... ....................... sensitisus (Graeter et Chappuis)

4 (3) Réceptacle séminal elliptique; branches furcales plus de 2,5 fois plus longues que larges; soies terminales internes et externes de longueur sensiblement analogues $\ldots \ldots \ldots \ldots \ldots \ldots \ldots \ldots . \ldots \ldots$

5 (6) Epines du dernier article de l'exopodite des $\mathrm{P}_{1}$ à $\mathrm{P}_{4}$ au nombre de $2-3-3-3 \ldots \ldots \ldots \ldots \ldots \ldots \ldots \ldots$ A. vernalis (Fischer)

6 (5) Ces épines au nombre de $3-4-4-4 \ldots \ldots \ldots$ A. robustus (Sars)

7 (8) Antennules à 12 articles ....................... 9

8 (7) Antennules à 11 articles; branches furcales glabres, 2,5 fois plus longues que larges; formule des épines externes des $\mathrm{P}_{1}$ à $\mathrm{P}_{4} 2-\mathbf{3}-3$

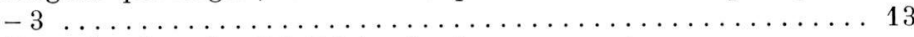

9 (10) Branches furcales 5 à 7 fois plus longues que larges $\ldots \ldots \ldots \ldots$ $\ldots \ldots \ldots \ldots \ldots \ldots \ldots \ldots$. capillatus (Sars)

10 (5) Branches furcales moins de 4 fois aussi longues que larges . ... 11

11 (12) Branches furcales ciliées intérieurement; aesthete du $9^{\mathrm{e}}$ article de l'antennule ne dépassant pas cet article; formule des épines des $\mathrm{P}_{1}$ à $\mathrm{P}_{4} 3-4-4-4 \ldots \ldots \ldots \ldots$. venustus (Norman et $\mathrm{S} \cot \mathrm{t}$ )

12 (11) Branches furcales glabres, trois fois plus longues que larges; aesthete du $9^{\mathrm{e}}$ article de l'antennule atteignant le $11^{\mathrm{e}}$ article; formule des épines des $\mathrm{P}_{1}$ à $\mathrm{P}_{4} 2-3-3-3$; espèce d'eaux souterraines $\ldots \ldots$. $\ldots \ldots \ldots \ldots \ldots \ldots \ldots \ldots \ldots \ldots \ldots$. rhenanus Kiefer 
13 (14) Soies furcales internes et externes subégales; espèce d'eaux souterraines ........................ kieferi (Chappuis)

14 (13) Soie furcale interne deux fois plus longue que l'externe; espèce d'eaux souterraines .................. hispanicus Kiefer

\section{AMPHIPODES}

Par F. Graf et R. Husson

C'est par centaines que des Crustacés Péracarides de l'ordre des Amphipodes étaient représentés dans les eaux du réservoir de la Fontaine des Suisses, lors de la visite de mai 1962. La grande majorité, cohabitant avec Niphargus virei Chevreux, appartenait à une petite espèce du genre hypogé Niphargus, maintes fois récoltée dans les puits, les grottes ou même le fond de certain lac, Niphargus kochianus kochianus Bate.

Cette espèce a été décrite il y a plus d'un siècle (1859) de GrandeBretagne par Spencer Bate d'après un unique exemplaire récolté dans l'eau d'un puits.

Une des particularités morphologiques de cette espèce, comme l'ont montrée les recherches du grand carcinologiste A. Schellenberg, est d'être avec $N$. laisi et $N$. inopinatus, une des rares espèces du genre montrant un développement à peu près identique des uropodes de la troisième paire chez les mâles et les femelles (ce qui a d'ailleurs poussé S. Karaman à rattacher cette espèce au genre Niphargopsis).

Comme une diagnose rapide du sexe est dans le genre en général basée sur un caractère sexuel secondaire très apparent, la différence de taille respective des deux articles terminaux des derniers uropodes, on a longtemps cru que dans cette espèce on ne récoltait que des femelles.

La détermination précise du sexe ne peut être basée avec certitude que sur la présence ou l'absence de pénis (Geschlechtspapillen) qui ne peut être décelée que par un examen attentif à la loupe binoculaire (difficile à faire sur le vivant étant donné la petitesse de l'espèce).

W. F. de Vismes Kane (1904) qui avait eu à sa disposition plus d'une centaine d'exemplaires, provenant des profondeurs d'un lac irlandais n'avait pu, malgré d'attentives recherches, déceler d'autres caractères morphologiques bien tranchés permettant de déterminer macroscopiquement le sexe, hésitant à trouver des critères sûrs, soit dans la mesure de la longueur des troisièmes uropodes qui ne paraissait guère être que proportionnelle à la taille de l'individu examiné, soit dans la position et le port du telson.

C'est Schellenberg qui le premier (1933) a montré qu'un critère valable pouvait être tiré de la conformation du gnathopode qui, si elle permettait comme on l'avait déjà remarqué, de caractériser 
l'espèce, pouvait présenter suivant le sexe des variations de taille et de forme.

L'espèce kochianus se caractérise en effet par un élargissement apical du propodite dont l'angle sterno-distal est nettement marqué, légèrement aigu et muni d'une forte épine, les bords adjacents étant plus ou moins concaves. Quant au mâle il se distingue de la femelle par la forme ainsi que par la taille du propodite, notamment en ce qui concerne le gnathopode 2 pour lequel le propodite est beaucoup plus ramassé et plus gros que dans le gnathopode 1 alors que chez la femelle les deux propodites ont à peu près la même taille.

La variation sexuelle est encore plus nette en ce qui concerne le carpopodite du deuxième gnathopode. En effet, si chez la femelle la longueur du carpopodite est plus grande que celle du propodite, le carpopodite d'un mâle adulte est plus court que le propodite du même appendice. De plus, pour les individus de même taille, le carpopodite du gnathopode 2 d'un mâle est moins développé que l'article correspondant d'une femelle. Ainsi, il est fort probable que chez $N$. kochianus kochianus il y ait énantiométrie de croissance pour le carpopodite des gnathopodes 2 du mâle comme cela a été montré chez l'Amphipode Talitridé Orchestia (H. Charniaux-Cotton 1957 - F. Graf 1958).

Il convient de plus de noter le dimorphisme sexuel que présentent les antennes qui sont plus développées chez le mâle (voir planche 52, B). Cette même photographie montre la taille comparable atteinte par les uropodes de la $3^{\mathrm{e}}$ paire dans les deux sexes. Par contraste la fig. A représente un mâle Niphargus aquilex aquilex montrant le développement presque hypertélique de ses uropodes terminaux. Ce dernier cliché est intéressant aussi en ce qu'il permet de bien distinguer les cinq paires de branchies qui sont insérées du deuxième au sixième segment du péréion.

L'étude du riche matériel niphargique récolté par l'hydrobiologiste belge R. Leruth avait amené la même année Schellenberg à distinguer plusieurs sous-espèces de kochianus et séparer notamment une sousespèce kochianus pachypus de la sous-espèce typique kochianus kochianus (la forme pachypus devant sa dénomination au fait que les articles des P. 7 sont trapus, le méropodite de ces derniers étant presque carré).

Pour les Niphargus kochianus signalés alors en France toutes les stations citées dans la Faune de France (1925) ont pour Schellenberg trait à la sous-espèce pachypus. La sous-espèce kochianus kochianus ne sera avec certitude citée en France qu'à la suite de la récolte qui en fut faite en décembre 1936 dans une flaque de la mine de fer abandonnée de Romange dans le Jura. (Hiermit ist die Unterart zum erstenmal mit Sicherheit auf französischem Gebiet nachgewiesen.) 
Depuis la récolte sus-mentionnée de 1936 la sous-espèce kochianus a été rencontrée dans plusieurs grottes ou puits aussi bien dans l'Est de la France que dans le Sud-Ouest ou les Pyrénées. D'une façon générale elle est connue de Grande-Bretagne, des Pays-Bas, de Belgique, de France, d'Allemagne (Rhénanie) et de Suisse (une seule station, la grotte du Chemin de fer, où elle a été découverte par Gigon 1957 en compagnie de $N$. foreli setiferus comme dans la mine de Romange d'où cette sous-espèce avait été décrite).

Il nous faut signaler que, outre les deux sous-espèces kochianus kochianus et kochianus pachypus on a décrit beaucoup d'autres sousespèces européennes:

N. kochianus irlandicus Schellenberg d'Irlande

N. kochianus polonicus Schellenberg de Pologne

$N$. kochianus wolfi Schellenberg de Yougoslavie

$N$. kochianus stygocharis Dudich de Roumanie

$N$. kochianus petrosani Dobreanu et Manolache de Bulgarie

N. kochianus longidactylus Ruffo d'Italie

Grace à l'abondante récolte (plusieurs centaines d'individus) de Niphargus kochianus kochianus faite à Dijon dans le réservoir de la Fontaine des Suisses nous avons pu constater que comme pour les exemplaires récoltés en Belgique par R. Leruth, la taille des mâles ne dépasse guère $5 \mathrm{~mm}$ et celle des femelles $5,5 \mathrm{~mm}$. Dans ce matériel pêché au mois de mai 1962, nous avons constaté qu'un grand nombre de femelles étaient ovigères vérifiant ainsi une fois de plus que, si dans le genre hypogé Niphargus les processus de reproduction se déroulent tout au long de l'année, ceux-ci présentent cependant un maximum de fréquence très net au printemps et au début de l'été comme on l'observe d'ailleurs chez d'autres Crustacés Péracarides des eaux souterraines comme les Asellus du groupe casaticus (J. P. Henry).

Le nombre des œufs par ponte étant d'une façon générale chez les Amphipodes, et en particulier dans le genre Niphargus, proportionnel à la taille atteinte par la femelle (Ginet 1960), il n'y a rien d'étonnant pour cette petite espèce à trouver un effectif peu élevé d'œufs embryonnés dans la poche incubatrice. Nous avons ainsi notamment observé : 6 femelles de $2,8 \mathrm{~mm}$ ne portaient chacune que 2 œufs (diamètre $480 \mu$ ) alors que 2 femelles de $3 \mathrm{~mm}$ avaient dans leur marsupium 5 œufs (diamètre de ceux-ci, pour l'une $450 \mu$, pour l'autre $470 \mu$ ) et que 2 femelles, ayant respectivement 3,7 et $4 \mathrm{~mm}$, avaient toutes deux 7 œufs de $420 \mu$ de diamètre.

L'effectif maximum d'œufs par portée dans cette sous-espèce semble donc être celui présenté par une femelle de Rhénanie (Düsseldorf) de 
$5 \mathrm{~mm}$ qui avaient 11 œufs, les autres femelles de cette station allemande ayant entre 5 et 8 oufs.

Les Niphargus récoltés en abondance à la Fontaine des Suisses appartiennent tous à la sous-espèce Niphargus kochianus kochianus de même que ceux (une trentaine) capturés en 1959 par l'un de nous dans le puits de la propriété du Médecin-Colonel Chabeuf à Mirebeau (20 km E. de Dijon).

Signalons que dans ce puits les Copépodes Cyclopides étaient nombreux et appartenaient (R. Rouch det. 1959) à l'espèce Acanthocyclops venustus, espèce que $\mathrm{B}$. Dussart (voir ci-dessus) a dénombré dans les Copépodes récoltés à la Fontaine des Suisses en signalant que ce Cyclopide n'était connu que de deux autres stations françaises (Alsace-Dauphiné).

On peut penser que la prospection méthodique des eaux souterraines (qui sera facilitée par l'usage du piège à Niphargus mis au point par F. Graf) aussi bien en Bourgogne que dans d'autres provinces aménera la découverte de nouvelles stations des deux sous-espèces françaises de Niphargus kochianus confirmant probablement le fait observé en Belgique par R. Leruth à savoir que c'est plutôt la sous-espèce kochianus kochianus que l'on rencontre de préférence et parfois en abondance dans les puits.

\section{RÉSUMÉ}

Inventaire des Crustacés récoltés en mai 1962 dans le réservoir de la Fontaine des Suisses à Dijon. Les Copépodes y sont représentés par cinq espèces: Macrocyclops albidus, Eucyclops serrulatus sous la forme typique et sous une forme légèrement différente du type, Eucyclops serrulatus var. mihi, Acanthocyclops venustus, Acanthocyclops vernalis et Acanthocyclops robustus. La coexistence de ces deux dernières formes dans un milieu aussi réduit que ce réservoir laisse à penser que nous avons bien là à faire à deux espèces bien distinctes. Une clé de détermination est donnée pour les espèces du genre Acanthocyclops.

Les Amphipodes y sont représentés par Niphargus virei et surtout Niphargus kochianus kochianus dont plusieurs centaines d'individus ont été recueillies. Au sujet de cette petite espèce, des considérations sont faites sur la répartition géographique, la ponte, le dimorphisme sexuel et les formes voisines.

\section{ZUSAMMENFASSUNG}

Inventar der im Mai 1962 im ,,Réservoir de la Fontaine des Suisses“ in Dijon gefangenen Crustaceen. Die Copepoden sind durch fünf Arten vertreten: Macrocyclops albidus, Eucyclops serrulatus (typische Form), Eucyclops serrulatus var. mihi, Acanthocyclops venustus, Acanthocyclops vernalis und Acanthocyclops robustus (leicht veränderte Form). Das gleichzeitige Vorhandensein der beiden letzten Formen in einem so verminderten Milieu läßt uns vermuten, daß wir es hier mit zwei gut getrennten Species zu tun 
haben. Wir geben einen Bestimmungsschlüssel für die Species der Gattung Acanthocyclops.

Die Amphipoden werden durch Niphargus virei und besonders durch Niphargus kochianus kochianus vertreten; davon wurden mehrere hundert Exemplare gefangen. Für diese kleine Art geben wir Angaben über die geographische Verbreitung, die Eiablage, den Dimorphismus der Geschlechter sowie über die benachbarten Formen.

\section{B I B LI O GRAPH I E}

\section{Gopépodes}

Dussart, B. (1964) - Copépodes d'Espagne. Bull. Soc. Zool. Fr. 89, 2/3 : 117-125.

Graeter, E. (1908) - Beiträge zur Kenntnis der schweizerischen Höhlenfauna. III. Ein neuer Höhlencopepode Cyclops crinitus nov. spec. Zool. Anz. $33:$ 45-49.

Gurney, R. (1933) - British Freshwater Copepoda. 3e Vol., 238 p. Cyclopoida, Caligoida. Dulau \& Co, Londres.

Herbst, H. V. (1953) - Liste der deutschen Calanoida und Cyclopoida Gnathostoma des Süßwassers. Schrift. Naturwiss. Vereins SchleswigHolstein 26, $2:$ 146-151.

Hertzog, L. (1930) - Notes sur quelques crustacés nouveaux pour la plaine d'Alsace. Bull. Assoc. Philomath. Alsace-Lorraine 7, $5: 355-364$.

Hutchinson, G. E. (1951) - Copepodology for the ornithologist. Ecol. 32, $3: 571-577$.

Kiefer, F. (1932) - Neue Süßwassercopepoden aus Jugoslawien. Zool. Anz. $101,3 / 4: 49-60$.

- (1936) - Über einige Ruderfußkrebse (Crustacea Copepoda) aus dem Grundwasser Belgiens. Bull. Mus. R. Hist. Nat. Belg. 12, $313 \mathrm{p}$.

- (1960) - Ruderfußkrebse (Copepoden). 1 Vol., 97 p. Kosmos-Verlag, Franckh, Stuttgart.

KLIE, W. (1928) - Cyclops venustus in Nordwestdeutschland. Zool. Anz. 78, $3 / 4: 62-66$.

Lindarerg, K. (1949) - Contributions à l'étude des Cyclopides (crustacés copépodes). Kungl. Fys. Sälls. Lund Förhandl. 19, 724 p.

- (1953) - Cyclopides (Crustacés copépodes) de la Turquie, en particulier comme habitants des grottes. Hidrobiol. Istambul B 1, 3: 149-185.

Lowndes, A. G. (1929) - The result of breeding experiments and other observations on Cyclops vernalis Fischer and Cyclops robustus G. O. Sars. Int. Rev. Hydrobiol. 21 : 171-188.

Ретколsкi, T. K. (1954) - Beitrag zur Kenntnis des jugoslawischen Cyclopiden. Acta Mus. Maced. Sci. Nat. 2, $1: 1-31$.

Roy, J. (1922) - Sur les Copépodes libres de la Côte d'Or. Bull. Soc. Zool. Fr. 47: 186-191.

SARs, G. O. (1913-1918): - An account of the crustacea of Norway. Vol. VI, 225p. - Copepoda cyclopoïda. Bergen.

Thallwit7, J. (1926) - Über Varietätenbildung bei Cyclops vernalis Fischer und Cyclops robustus Sars. Arch. Hydrobiol. 17: 366-380.

Thié вaud, M. (1953) - Les entomostracés de la Vieille-Thiele à Meienried et des environs de Bienne. Rev. Suisse de Zool. 60, 4 : 567-614. 
Amphipodes

Aellen, V., et Gigon, R. (1964) - La grotte du chemin de fer. Bull. Soc. Neuchatel Sc. Nat. 87: 5-25.

Charniaux-Cotton, H. (1957) - Croissance, régénération et déterminisme endocrinien des caractères sexuels secondaires d'Orchestia gammarella (Pallas), Crustacé Amphipode. Ann. Sc. Nat., Zool., 11e série, 19: 411-560.

Chevreux, E., et Fage, L. (1925) - Amphipodes. Faune de France, 9, 488 pp. Lechevalier, Paris.

Ginet, R. (1960) - Ecologie, éthologie et biologie de Niphargus (Amphipodes Gammaridés hypogés). Ann. Spéléo. 15: 239-376.

Graf, F. (1958) - Développement post-embryonnaire des gonades et des glandes androgènes d'Orchestia cavimana (Heller), Crustacé Amphipode. Bull. Soc. Sc. Nancy 17 : 233-261.

- (1966) - Description d'un piège à Niphargus. International Journal of Speleology. 2 : 287-289.

Henry, J. P. (1965) - Contribution à l'étude de la biologie d'Asellus cavaticus Leydig. International Journal of Speleology $1: 279-286$.

Husson, R. (1939) - Amphipodes des galeries de mines de France. Arch. Zool. expér. géné. $81: 101-111$.

Leruth, R. (1938-1939) - Notes d'hydrobiologie souterraine. Remarques écologiques sur le genre Niphargus 1 et 2. Bull. Soc. Roy. Sciences Liège.

SchellenberG, A. (1933) - Die Niphargen des Brüsseler naturhistorischen Museums. Bull. Mus. Roy. Hist. Nat. Belgique 9, $50: 8$.

- (1933) - Höhlenflohkrebse des Adelsberger Grottensystems nebst Bemerkung über Niphargus kochianus. Mitt. Höhl.-Karstforsch. 32-37.

- (1937) - Bemerkungen zu meinem Niphargus-Schlüssel und zur Verbreitung und Variabilität der Arten, nebst Beschreibung neuer NiphargusFormen. Mitt. Zool. Mus. Berlin 22: 1-44.

- (1937) - Niphargen (Amphipoda) des Französischen Jura und Jugoslawiens. Zool. Aus. 120:161-169.

Sтоск, J. H. (1961) - Ondegrondse waterdieren. Zuid. Limburg. Natuurhst. Maandbl. 77-85.

de Vismes Kane, W. F. (1904) - Niphargus kochianus Bate in an irish lake and N. subterraneus Leach in Kent. Ann. Mag. Natur. Hist. London 14: $274-283$.

\section{LÉGENDES DES PLANCHES 50 (1) - 52 (3)}

Fig. 1-6: Eucyclops serrulatus - 1: forme typique, vue générale d'une femelle; 2 : furca; $3: \mathrm{P}_{5} ; 4$ : Endopodite 3 de $\mathrm{P}_{4} ; 5: \mathrm{P}_{5}$ anormale; 6 : vue ventrale des $\mathrm{Th}_{5}$ et $\mathrm{Abd}_{1}$.

Fig. 7, 8: Acanthocyclops venustus $-7: \mathrm{P}_{5} ; 8:$ furca, vue ventrale.

Fig. 9-14: Derniers articles des $\mathrm{P}_{4}$ de plusieurs formes d'Acanthocyclops robustus (a: endopodite; b: exopodite) - soit 9: Camargue, première forme ; 10: Lac d'Eguzon (Creuse) ; 11 : Las Marismas (Espagne); 12: Fontaine des Suisses à Dijon; 13: Camargue, deuxième forme; 14: Etang du Plessis (Loir et Cher).

A. - Niphargus aquilex aquilex (grotte d'Antheuil, Côte d'Or). Mâle de $22 \mathrm{~mm}$. 
B. - Niphargus kochianus kochianus - à gauche $=$ femelle portant 3 œufs ; - à droite $=$ mâle de $4 \mathrm{~mm}$.

C. - Gnathopodes 1 et 2 d'une femelle de $N$. kochianus kochianus.

D. - Gnathopodes 1 et 2 d'un mâle de $N$. kochianus kochianus. 


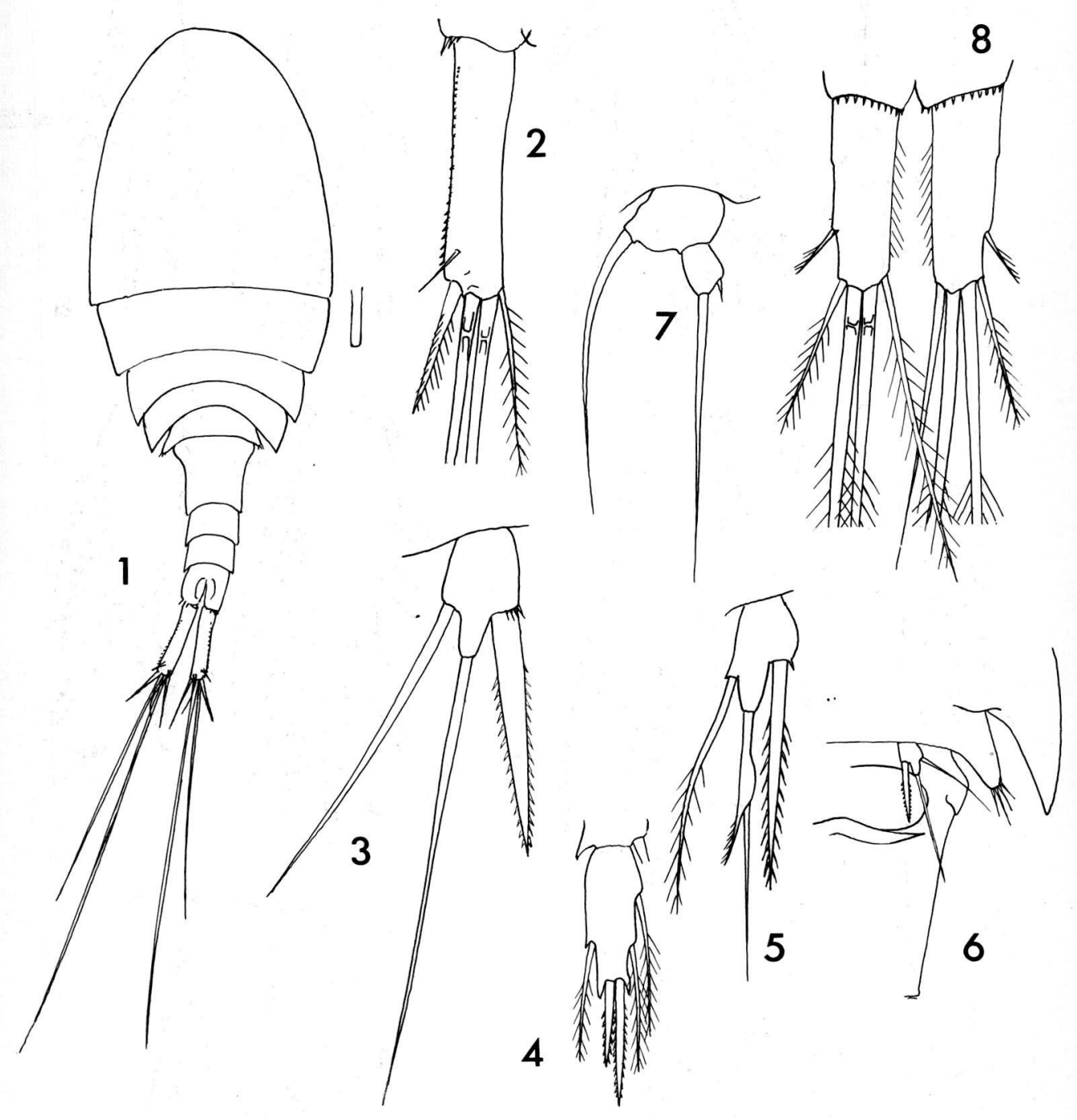



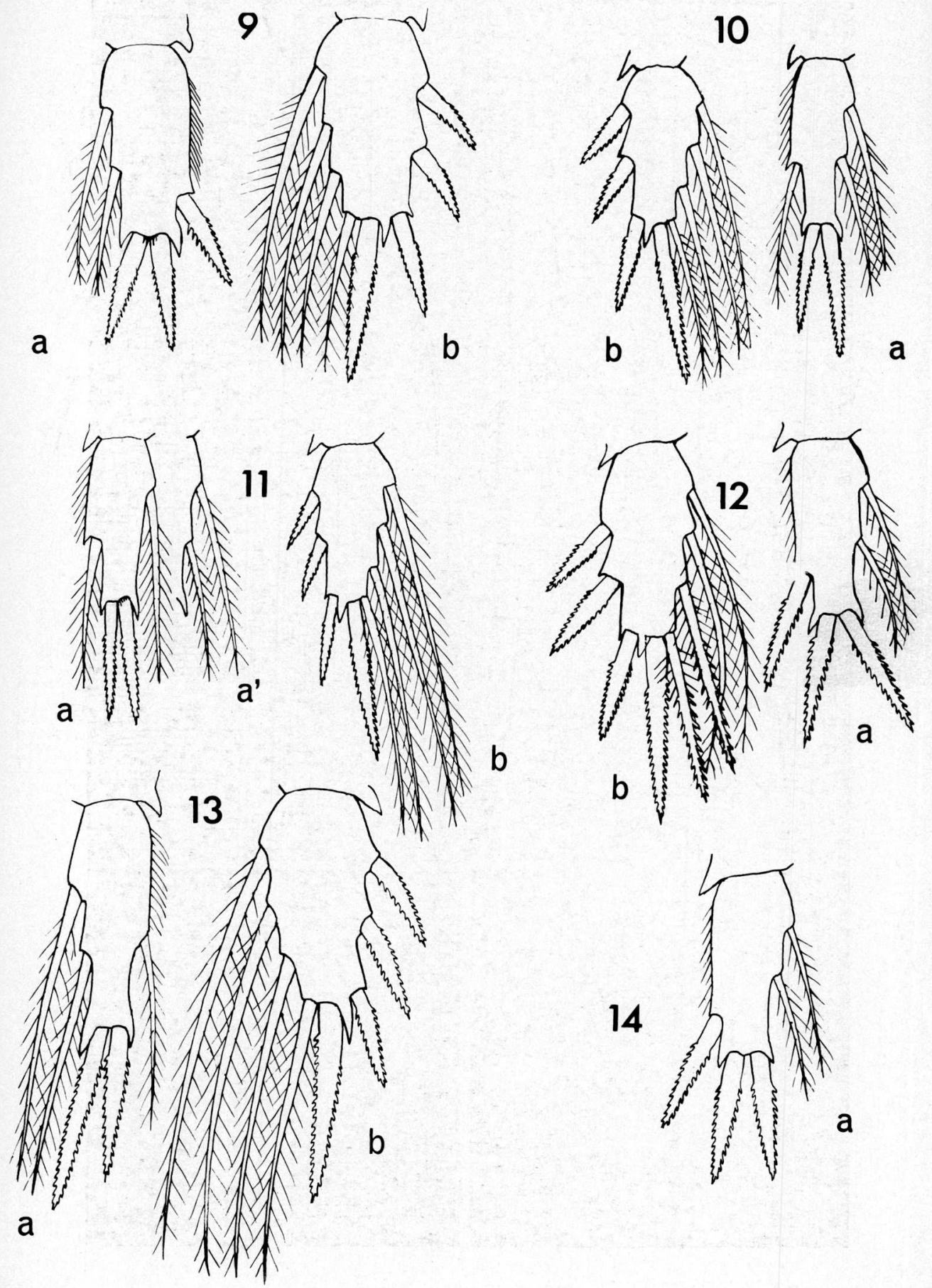

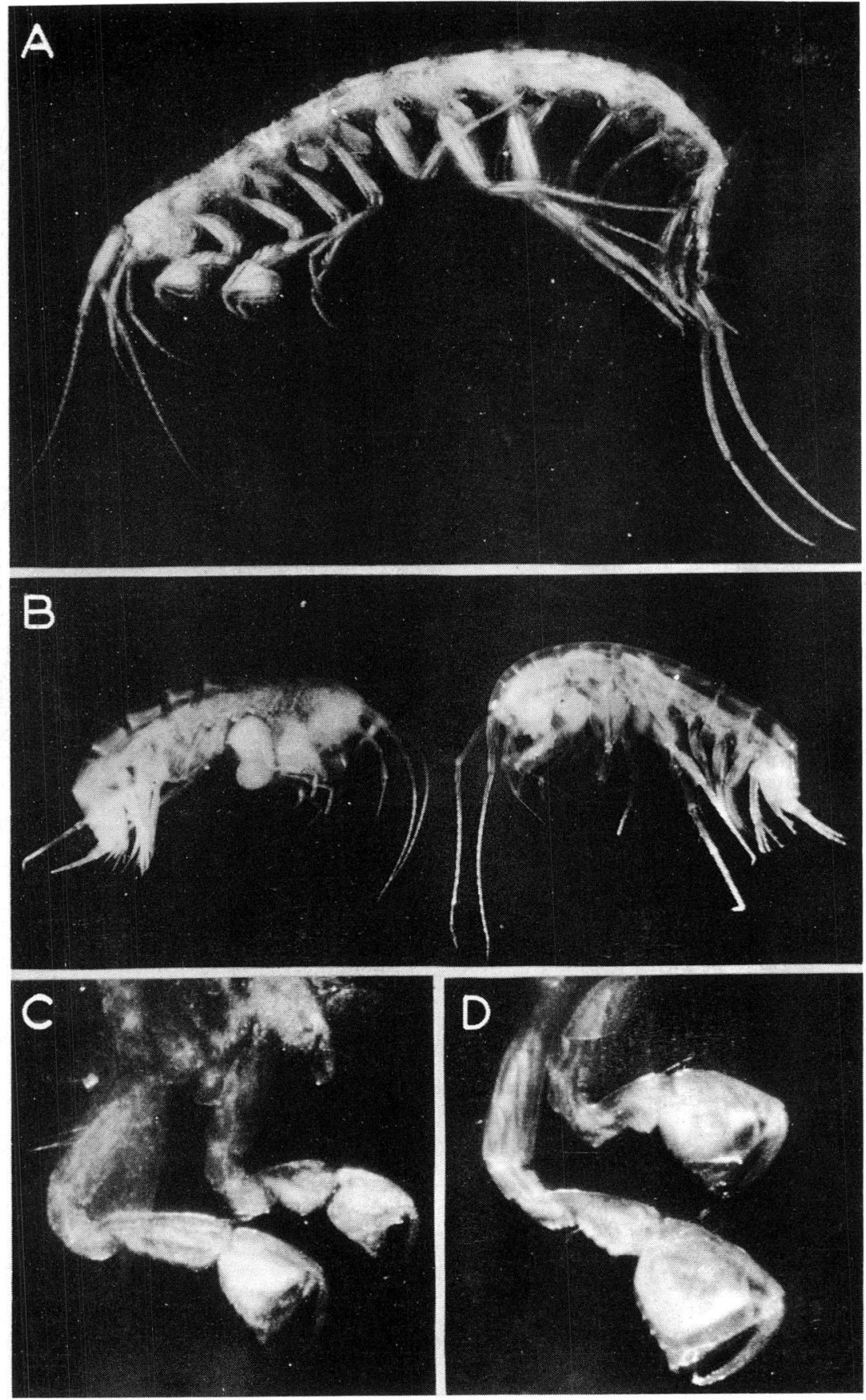